\title{
Servicios y Aparatos para el Control de Mosquitos en Viviendas de Florida. ${ }^{1}$
}

Jorge R. Rey y C. Roxanne Rutledge ${ }^{2}$

\section{¡Sea un Consumidor Educado!}

Existen muchos aparatos en el mercado que pretenden ser efectivos para el control de mosquitos. Los anuncios para estos aparatos enfocan al típico consumidor. ¿Como se puede averiguar si estos aparatos en verdad son efectivos?

Desafortunadamente, muchos comerciantes se aprovechan del afán que tenemos de proteger a nuestras familias contra las enfermedades transmitidas por mosquitos. Ofrecemos este artículo para ayudar al consumidor a llegar a decisiones inteligentes en cuanto a la protección de su salud y la de su familia.

\section{Control de Mosquito y la Prevención de Enfermedades Acarreadas por Mosquitos}

Control de mosquitos y prevención de enfermedades acarreadas por mosquitos no son la misma cosa. El control de mosquitos consiste en la reducción de las poblaciones de mosquitos, lo cual posiblemente puede efectuar una reducción en el número de picadas por mosquitos en ciertas áreas. Prevención de enfermedades acarreadas por mosquitos significa protección personal, incluyendo el uso de repelentes adecuados. ¿Cual es la diferencia entre las dos? La meta del control de mosquitos no es eliminar todas las picadas de mosquitos, sin embargo, la prevención de enfermedades acarreadas por mosquitos significa que cada individuo toma la responsabilidad de protegerse de cualquier picada de mosquito.

Los equipos y servicios mencionados en este artculo no están diseñados para la prevención de enfermedades; estos productos son usados para el control de mosquitos.

\section{Trampas para Mosquitos}

Muchos tipos de trampas para mosquitos están basadas en la generación de dióxido de carbono $\left(\mathrm{CO}_{2}\right)$ para atraer a los mosquitos al aparato. Cuando los mosquitos se encuentran cerca del aparato, un ventilador los succiona al recipiente de

1. Este documento con la identificación: ENY-692S, es uno de una serie de publicaciones del Departamento de Entomology and Nematology, del Servicio de Extensión Cooperativo de la Florida, del Instituto de Alimentos y Ciencias Agrícolas, universidad de la Florida. Publicado por primera vez en Febrero, 2004. Por favor, visite la dirección en la Red EDIS en 〈http://edis.ifas.ufl.edu〉.

2. Jorge R. Rey, professor and Roxanne Rutledge assistant professor, Entomology and Nematology Department, Florida Medical Entomology Laboratory, Vero Beach, Cooperative Extension Service, Institute of Food and Agricultural Sciences. University of Florida, Gainesville, FL 32611

El uso de nombres comerciales que han sido mencionados en esta publicación fue únicamente con el propósito de proporcionar información específica. El hecho de nombrar estos productos por UF/IFAS, no es una garantía o garantiza que sean los más efectivos en el mercado, y no significa que son avalados por la institución y que otros compuestos similares deben ser excluidos. 
colección, donde los mosquitos mueren. La inversión inicial para uno de estos aparatos varía de $\$ 300$ a $\$ 1,400$.

Estas trampas con "carnada de $\mathrm{CO}_{2}$ " pueden atrapar a muchos mosquitos, pero una colección impresionantemente grande, que llene el recipiente, puede representar solo una minucia de todas la hembras en busca de sangre que existen en las cercanías, lo cual significa que en estos casos, el uso de la trampa no resultará en una diminución significativa en las poblaciones de mosquitos. No existen en estos momentos ningunos datos científicos que indiquen que estos aparatos son efectivos en el control de mosquitos, en la reducción de poblaciones de mosquitos, o en la reducción de las tasas de picadas por mosquitos bajo las condiciones que normalmente se encuentran en diferentes viviendas. Hasta que no exista evidencia científica, uno debe evitar depender demasiado en la capacidad de estos aparatos para controlar mosquitos en su vivienda.

Algunos anuncios indican que estas trampas aniquilan las poblaciones de mosquitos en cuatro a seis semanas. El palmo de vida de los mosquitos adultos varía dependiendo de las especies y de la temperatura ambiental, pero las poblaciones de muchos mosquitos normalmente diminuyen naturalmente en varias semanas sin intervención humana, particularmente si las lluvias son intermitentes, y si el área está sujeta a medidas de control profesionales. No existe evidencia alguna que estas trampas causan disminución de las poblaciones de mosquitos.

Existen circunstancias bajo las cuales los aparatos para atrapar mosquitos pueden reducir las tasas de picadas por mosquitos en áreas pequeñas durante cortos periodos de tiempo. Varios factores tienen que ser óptimos para que esto suceda:

- Debe haber poco viento que interfiera con la nube de $\mathrm{CO}_{2}$.

- La abundancia de mosquitos debe ser baja desde el principio.

- La nube atrayente de $\mathrm{CO}_{2}$ debe ser lo suficientemente grande para que gane la competencia con otros atrayentes, por ejemplo la atracción por grupos de personas o animales.

Como es con otros productos, tener cuidado es buen consejo. La única información disponible sobre cuan bien funciona estos aparatos son los testimonios de aquellos que los han comprado. Dichos testimonios no son el resultado de estudios controlados ni de análisis adecuados de datos.

\section{Sistemas Programados de Rociado de Insecticidas}

Un sistema programado de rociado es un servicio que el consumidor puede comprar para matar mosquitos. El sistema incluye inyectores para el rociado de insecticidas conectados a tuberías que se instalan en el perímetro de la vivienda. Las tuberías se conectan a un tanque de insecticida (de 115 a 965 litros); el rocío de insecticidas se controla por reloj.

Para poder reducir el número de mosquitos de cualquier especie, uno debe monitorear varias variables y tomar las medidas de control apropiadas, las cuales son específicas para las diferentes especies. Esto constituye la ciencia (y el arte) de vigilancia para mosquitos. La vigilancia debe incluir:

- Identificación correcta de las especies que causan el problema.

- Consideración del comportamiento de las diferentes especies.

- Evaluación de la densidad poblacional; conteos de las tasas de aterrizaje; conteos en trampas; desarrollo de las larvas.

- Monitoreo de las condiciones ambientales.

¿Porqué es importante la identificación

correcta de las especies de mosquitos?

- Programas efectivos y eficientes para el control de mosquitos responden a la densidad de mosquitos. No es apropiado aplicar insecticidas para matar a los mosquitos adultos si no hay mosquitos adultos en el área durante la aplicación. 
- Es crítico determinar el momento apropiado para la aplicación. Puede ser muy difícil decidir cual es el momento apropiado para aplicar adulticidas específicamente contra mosquitos que están reposando o que están al ala.

- Cualquier aplicación de insecticida que depende en aplicaciones programadas sin basarse en la vigilancia y en decisiones hechas por humanos resulta en aplicaciones no apropiadas. Este tipo de aplicación puede contribuir al desarrollo de la resistencia a los insecticidas en los insectos, y puede causar problemas ambientales.

Sistemas programados de rociados de insecticidas, o cualquier otro sistema que simplemente aplica insecticidas a intervalos de tiempo, ya sea para formar una barrera contra los mosquitos, o para matar a los mosquitos que están al ala, no incluyen el elemento humano el cual es crítico para el control efectivo y ambientalmente sano. La promoción de aplicación indiscriminada de insecticidas basada solamente en intervalos de tiempo va contra todas las reglas del control eficiente y responsable de los mosquitos.

\section{Aparatos Ultrasónicos}

Los aparatos ultrasónicos son productos que están diseñados para usarse en la muñeca, alrededor del cuello, o fijados a un cinturón, para repeler a los mosquitos. Estos aparatos emiten sonidos que imitan a los de los mosquitos machos o a los de caballitos del diablo (libélulas) y teoréticamente asustan a los mosquitos hembras. Estas pretensiones no tienen base alguna en evidencia científica. Mosquitos hembras en busca de una cena de sangre no huyen de los mosquitos machos, y ni las hembras ni los machos evitan áreas donde las libélulas están presentes.

En agosto del 2002 la Comisión Federal de Comercio (Federal Trade Comission - FTC) acusó a una compañia de Florida de emitir afirmaciones falsas y no probadas sobre sus línea de productos MosquitoContro* . Dichos productos, los cuales son operados por baterías, incluyen uno usado como brazalete, un llavero, y un modelo fijo. De acuerdo al
FTC, no existe evidencia competente o confiable que apoye las afirmaciones sobre estos productos y las afirmaciones son falsas.

*Sumario de Procedimiento Legal No. 9303, en el caso de Lentek International, Inc., Joseph Durek y Lou Lentine. Archivo del FTC número 012-3117.

\section{Incineradores de Insectos - "Bug Zappers"}

Estos productos usan la luz ultravioleta para atraer a los mosquitos a trampas que los electrocutan. Estos aparatos atraen también a muchos otros tipos de insectos, incluyendo a las mariposas y a los escarabajos. Estudios científicos indican que estos aparatos no reducen las poblaciones de mosquitos, y que matan a otros tipos de insectos con mucha mas frecuencia que a los mosquitos.

\section{Murciélagos y Golondrinas de Iglesia}

Los murciélagos y las golondrinas de iglesia (Progne subis) comen mosquitos; sin embargo, como casi todos los otros organismos, ellos tienen una dieta variada. Las especies que dependen exclusivamente en una sola fuente de alimento pueden ser eliminadas rápidamente si hay escasez o falta completa de esta fuente.

Los mosquitos constituyen solo una pequeña porción de la dieta de murciélagos y aves. No existe evidencia alguna de que ningún murciélago o ave pueda controlar a los mosquitos cuando los útimos son abundantes.

No es prudente, especialmente cuando el riesgo de exposición a enfermedades transmitidas por mosquitos es alto, depender en aves o murciélagos para controlar los mosquitos. No cabe duda de que consumirán algunos mosquitos, pero no en números suficientes para efectuar una reducción apreciable en el número de mosquitos.

\section{Observaciones Adicionales}

"Natural" es una palabra que recientemente se ha usado para promover productos sanos y seguros. Desafortunadamente, este vocabulario puede engañar 
individuos no bien informados. Productos hechos o derivados de algo natural no son necesariamente seguros y sanos. Recuerde que la sal es natural, pero puede ser dañina a la salud. Además, productos seguros o naturales pueden no ser efectivos.

Anuncios para aparatos para el control de los mosquitos que afirman que reducen el virus del Nilo Occidental $u$ otras enfermedades acarreadas por mosquitos no son apropiados y no dicen la verdad. No se ha probado que exista algún aparato que tenga el mas mínimo impacto en la reducción de la transmisión de enfermedades acarreadas por mosquitos.

Eliminar los criaderos de mosquitos cerca de la casa es un componente importante de cualquier programa para el control de los mosquitos. Para más información visite las páginas web http://fmel.ifas.ufl.edu y http://eis.ifas.ufl.edu.

Los repelentes que contienen DEET son los más efectivos para la protección personal contra las picadas de mosquitos. Para más informacin sobre como protegerse contra las picadas de mosquitos, vea la hoja informativa IN-419S, "Repelentes Contra Mosquitos".

\section{Referencias y Lecturas Adicionales}

Kale, II, H. W., 1968. The Relationship of Purple Martins to Mosquito Control. The Auk. 85(4):

654-661.

Nasci, R., C. W. Harris, and C. K. Porter. 1983. Failure of an Insect Electrocuting Device to Reduce Mosquito Biting. Mosquito News. 43(2): 180-184.

Oi, F. M. and R. Anderson. 2003. Mosquitoes In and Around the Home. EDIS. ENY-2004.

Rey, J. R. y Rutledge, C. R 2003. Repelentes contra Mosquitos. EDIS. IN419S. 